\title{
Performance of Mattis dementia rating scale-Chinese Version in patients with mild cognitive impairment and Alzheimer's disease
}

\author{
Shuxia QIAN \\ the second affiliated hospital of Jia Xing University \\ Keliang CHEN \\ Huashan Hospital Fudan University Department of neurology \\ Qiaobing GUAN \\ department of neurology,the second affiliated hospital of Jiaxing University \\ Qihao Guo ( $\nabla$ qhguo@sjtu.edu.cn ) \\ Shanghai Jiao Tong University affiliated six people's hospital
}

\section{Research article}

Keywords: Alzheimer's disease, mild cognitive impairment, Mattis Dementia Rating Scale

Posted Date: March 9th, 2020

DOI: https://doi.org/10.21203/rs.3.rs-16409/v1

License: (c) (i) This work is licensed under a Creative Commons Attribution 4.0 International License. Read Full License 


\section{Abstract}

Objective: To identify the applicability of the Chinese Version of Mattis Dementia Rating Scale (DRS-CV).

Methods: The DRS-CV was administered to 483 participants, including 136 normal controls, 167 patients with mild cognition impairment (MCl), and 180 patients with Alzheimer's disease (AD). Receiver Operating Characteristic (ROC) curve was used to evaluate the sensitivity and specificity of the scale.

Results: The scores of DRS-CV were ranked in the order of $\mathrm{NC}>\mathrm{MCl}>$ mild $A D>$ moderate $A D$ group. Memory was the sensitive function affected at a relatively earlier stage of AD. ROC curve analysis indicated the DRS-CV total score and memory subscale showed excellent sensitivity and specificity in the discrimination between $\mathrm{MCl}$ from mild $A D$ and mild $A D$ from moderate $A D$, but poor sensitivity and specificity in the discrimination between $\mathrm{MCl}$ and $\mathrm{NC}$.

Conclusion: The DRS-CV is useful to the early diagnosis and severity of $A D$, not to the early identification of $\mathrm{MCl}$.

\section{Introduction}

The Mattis Dementia Rating Scale (DRS) was used as a screening tool to assess the cognitive function for patients with dementia. It consists of 37 tasks divided into five subscales of attention, initiation/perseveration, construction, conceptualization, and memory, with subscale scores of $37,37,6,39,25$, respectively and a maximum total score of 144 .

The DRS provides more detailed information about a patient's impaired and intact cognitive function than other widely used mental state examination tools, such as the Mini-mental state examination (MMSE). The pattern of DRS scores obtained on the five domains can show qualitative differences in the cognitive profiles of different types of dementia, including Parkinson's disease (PD) [1], Huntington's disease [2], Lewy Body dementia [3] and Progressive supranuclear palsy [4]. Recently, the scale has been appled to a wider range disorders, such as mild cognitive impairment (MCl) due to PD, and schizophrenia [5].

Some sociodemographic factors, such as age and education, have been found to have a significant impact on the performance of DRS[6], and cross-cultural comparisons of the performance of instruments are often needed before generalisations can be made to apply them to people from different cultures. The scale was translated into Chinese, and the test-retest and inter-rater reliability of Chinese Version of the Dementia Rating Scale (DRS-CV) was excellent [7]. Since the DRS-CV has not yet been adoped nationwide, relavant studies have been conducted only in Hong Kong[8, 9]. The study sample was relatively small and the identification of cognitive impairment was not detailed. Our study, for the first time, validates Mandarin Chinese version of DRS and further assesses the applicability of DRS.

\section{Methods}

\subsection{Participants}

One hundred and thirty-six normal controls (NC) were recruited from the urban centers of Jingansi district of Shanghai. Controls had no known neurological or psychiatric diseases and were designated to be normal on the Clinical Dementia Rating Scale (CDR = 0 ) [10] and Mini-mental state examination-Chinese version (CMMSE score[cutoff) [11].

One hundred and sixty-seven patients with $\mathrm{MCl}$ and one hundred and eighty patients were recruited from Memory Clinic of Huashan Hospital.

The inclusion criteria for $\mathrm{MCl}$ [12] included the following: (1) cognitive impairment verified by an agent or caregiver; (2) symptoms last for more than 3 months; (3) between the ages of 50 and 90; (4)the lenth of schooling from 2 to 18 years; (5) the total score of CMMSE[cutoff [11], abnormal objective cognitive impairment documented by scoring below the age and 
education adjusted cutoff on the Neuropsychological assessments (Auditory verbal learning test) [13], preserved basic activities of daily living/minimal impairment in complex instrumental functions; (6) unknown etiology, (7) normal sense of hearing and sight; (8) dementia diagnostic criteria that do not meet the diagnostic criteria of the national institute on aging (NIA) and the alzheimer's association (AA)[14].

The diagnosis of AD is based on the NIA-AA standard diagnostic guidelines[14]. All patients were screened in detail including the history of brain disease, physical illness, and mental states such as anxiety and depression. In addition, all patients underwent neurological examinations, thyroid function tests ( $\mathrm{FT}_{3}, \mathrm{FT}_{4}$, and TSH), folic acid, and vitamin $\mathrm{B} 12$ measurements. CT or MRI scan of brain were conducted for all patients to exclude vascular factors (including lacunar infarctions or diffuse white matter ischemic changes). According to $C D R$, there were 116 cases of mild dementia $(C D R=1)$ and 64 cases of moderate dementia $(\mathrm{CDR}=2)[10]$.

This study was approved by the ethics committee of Huashan Hospital, and written consent was obtained from subjects or their legally authorized caregivers.

\subsection{Neuropsychological assessment}

The DRS-CV and CMMSE were used. The CDR was supported by participant and caregiver interview. Meanwhile, participants from Memory Clinic were also administered a battery of neuropsychological tests, including Auditory verbal learning test (AVLT), ${ }^{14}$ Logical memory test (LMT), ${ }^{15}$ Rey-Osterrieth Complex Figure Test (CFT), ${ }^{16}$ Clock-drawing test (CDT), ${ }^{17}$ Verbal fluency test (VFT), ${ }^{18}$ Stroop color-word test (SCWT), ${ }^{19}$ Trail making test (TMT) ${ }^{20}$ and Center for Epidemiology Scale-Depression (CES-D). ${ }^{21}$ All neuropsychological battery was administered by clinical psychologists or specially trained doctoral level students who were blinded to diagnosis.

\subsection{Statistical Analysis}

The $\chi 2$ test or one-way analysis of variance was used to test for the differences among the four groups (NC, MCl, mild $A D$ and moderate $A D$ groups) in the distribution of gender, age, education level and performance on neuropsychological tests. Bivariate correlation were applied to analyze the relation between two variables. Receiver Operating Characteristic (ROC) curve was used to evaluate the sensitivity and specificity of the scale. The level of significance was set at $a=0.05$. All analyses were conducted using SPSS version 19.0.

\section{Results}

\subsection{Demographic Characteristics and performance of CMMSE and DRS-CV}

Demographic information and the scores of CMMSE and DRS-CV among four groups including NC, $\mathrm{MCl}$, mild $\mathrm{AD}$ and moderate $A D$ were presented in Table 1. No difference was found in age $(F=2.428, p=0.065)$, sex distribution $(X 2=0.647$, $p=0.886)$ and education $(F=1.500, p=0.214)$ among the four groups.

The total scores of CMMSE and DRS-CV were significantly lower in patient groups. The scores of different cognitive domains were ranked in the order of $\mathrm{NC}>\mathrm{MCl}>$ mild $\mathrm{AD}>$ moderate $\mathrm{AD}$ group. Subscales of conceptualization and memory was affected at a relatively earlier stage.

\subsection{Correlations between demographic variables and DRS total and subscale scores}

We analyzed the effects of age, sex and education on DRS-CV total and subscale scores for NC. There was no difference on DRS-CV total and subscale scores between male and female (all $p>0.05$ ). Correlation analysis revealed that age was correlated to DRS-CV total score $(r=-0.264, p=0.002)$ and initiation/perseveration score $(r=-0.223, p=0.009)$, and education was correlated to DRS-CV total score $(r=0.254, p=0.003)$, attention score $(r=0.363, p<0.001)$ and initiation/perseveration score $(r=0.271, p=0.001)$. 
3.3 ROC analysis of DRS-CV total and subscale scores for discriminating $\mathrm{MCl}$ from $\mathrm{NC}, \mathrm{MCl}$ from mild $\mathrm{AD}$ and mild $\mathrm{AD}$ from moderate AD.

The most appropriate cutoffs of DRS-CV were 131 in the discrimination between $\mathrm{MCl}$ and NC (sensitivity $65.3 \%$, specificity 67.6\%, AUC 0.708), 120 in the discrimination between $\mathrm{MCl}$ and mild AD (sensitivity $84.5 \%$, specificity $85.0 \%, A U C$ 0.924), 103 in the discrimination between mild and moderate AD (sensitivity 79.7\%, specificity 78.4\%, AUC 0.846). Using these cutoff scores, the DRS-CV showed excellent sensitivity and specificity in the discrimination between $\mathrm{MCl}$ from mild $A D$ and mild $A D$ from moderate $A D$, but poor sensitivity and specificity in the discrimination between $\mathrm{MCl}$ and $\mathrm{NC}$, suggesting that the predictive information captured by the DRS total score was only reasonably good to detect AD but not $\mathrm{MCl}$.

The memory subscale showed good sensitivity and specificity in the discrimination between $\mathrm{MCl}$ from mild $A D$ and mild $A D$ from moderate $A D$, similar to the DRS total score. In comparison, the AUC of the memory subscale for discriminating $\mathrm{MCl}$ from mild $A D$ was similar to the initiation/perseveration subscale $(0.885$ versus $0.845, p=0.201)$ and larger than other three subscales (attention: 0.885 versus $0.681, p<0.001$; construction: 0.885 versus $0.605, p<0.001$; conceptualization: 0.885 versus $0.695, p<0.001)$. For discriminating mild $A D$ from moderate $A D$, the $A U C$ of the memory subscale was lager AUC than other four subscales, but not significant (attention: 0.823 versus $0.740, p=0.100$; initiation/perseveration: 0.823 versus $0.755, p=0.179$; construction: 0.823 versus $0.720, p=0.051$; conceptualization: 0.823 versus $0.670, p=0.005$ ). All DRS-CV subscale scores had poor sensitivity and specificity for discriminating MCI from mild AD.

\section{Discussion}

Screening for $A D$ is an important clinical necessity for early diagnosis and initiating proper treatment. The DRS is a psychometric instrument designed to assess the nature and severity of dementia. Five subscales are included to evaluate different domains of cognitive function.

In our study, we evaluated the performance of DRS-CV in different stages of $A D$, including very early stage (MCI). At the early stage of dementia, memory and conceptualization were first impaired. With progression of the disease, other functions such as initiation/perseveration and attention may be affected. ROC curve analysis indicated DRS-CV could be administered to differentiate AD from $\mathrm{MCl}$, but was not effective for detecting $\mathrm{MCl}$. Meanwhile, it is important to establish whether each of the five DRS subscales contributes equally to the diagnostic power. The result of ROC analysis showed the memory subscale might be better than other four subscales.

To our knowledge, several studies explored the effect of age and education on the DRS-CV score. Age and education conversion scores were obtained from researchers associated with the Mayo Older American Normative Studies (MOANS) [15]. Five equations to adjust for the age and educational level of the scores were provided [9]. In our study, age and educational level were related to the performance of DRS, consistent with previous studies.

We found the initiation/perseveration score of NC sample in our study was inferior to the studies in western countries and Hong Kong. Possible differences in lifestyle and culture might account for those differences. Initiation/perseveration subscale of DRS-CV included five tests: Supermarket Fluency, Clothing Fluency, Verbal Repetition, Double Alternating, and Graphomotor. For example, supermarkets were not ubiquitous in most cities of China until recent years, which may be a reason for low scores of that test in the Chinese elderly population.

Overall, our study verified the applicability of DRS-CV to detect AD but not $\mathrm{MCl}$. Our study was limited by several factors. There were no subjects with severe dementia $(C D R=3)$. The percentage of different stages was not evenly distributed. Subjects with low level of education and uneducated were not recruited adequately. Further investigation is required to confirm these results and to determine whether the cutoff scores are suitable in patients with lower educational level.

\section{Conclusion}


The DRS-CV is useful to the early diagnosis and severity of AD, not to the early

\section{Abbreviations}

DRS Mattis Dementia Rating Scale

DRS-CV Chinese Version of the Dementia Rating Scale

$A D$ Alzheimer's disease

$\mathrm{MCl}$ mild cognitive impairment

ROC Receiver Operating Characteristic

MMSE Mini-mental state examination

PD Parkinson's disease

NC normal controls

CMMSE Mini-mental state examination-Chinese version

AVLT Auditory verbal learning test

LMT Logical memory test

CFT Rey-Osterrieth Complex Figure Test

CDT Clock-drawing test

VFT Verbal fluency test

SCWT Stroop color-word test

TMT Trail making test

MOANS Mayo Older American Normative Studies

\section{Declarations}

\section{Ethics approval and consent to participate}

This study was approved by the ethics committee of Huashan Hospital, and written consent was obtained from subjects or their legally authorized caregivers.

\section{Consent for publication}

The authors consent to the publication of this article.

Availability of data and materialThe datasets used or analysed during the current study are available from the corresponding author Qihao Guo on reasonable request.

\section{Competing interests}

We state that there is no conflict of interest in this article. 


\section{Funding}

This work was supported by the Natural Science Foundation of China (number: 30570601).

\section{Authors' contributions}

Shuxia Qian did the scale assessment,collected the data and did statistical analysis.Keliang Chen wrote the paper. Qiaobing Guan and Qihao Guo designed the study and performed the research.

\section{Acknowledgements}

None.

\section{References}

1 Pontone GM, Palanci J, Williams JR, Bassett SS: Screening for DSM-IV-TR cognitive disorder NOS in Parkinson's disease using the Mattis Dementia Rating Scale. International journal of geriatric psychiatry 2013;28:364-371.

2 Paulsen JS, Butters N, Sadek JR, Johnson SA, Salmon DP, Swerdlow NR, Swenson MR: Distinct cognitive profiles of cortical and subcortical dementia in advanced illness. Neurology 1995;45:951-956.

3 Connor DJ, Salmon DP, Sandy TJ, Galasko D, Hansen LA, Thal LJ: Cognitive profiles of autopsy-confirmed Lewy body variant vs pure Alzheimer disease. Archives of neurology 1998;55:994-1000.

4 Rosser AE, Hodges JR: The Dementia Rating Scale in Alzheimer's disease, Huntington's disease and progressive supranuclear palsy. Journal of neurology 1994;241:531-536.

5 Bezdicek O, Michalec J, Nikolai T, Havrankova P, Roth J, Jech R, Ruzicka E: Clinical validity of the Mattis Dementia Rating Scale in differentiating mild cognitive impairment in Parkinson's disease and normative data. Dementia and geriatric cognitive disorders 2015;39:303-311.

6 Foss MP, Vale Fde A, Speciali JG: [Influence of education on the neuropsychological assessment of the elderly: application and analysis of the results from the Mattis Dementia Rating Scale (MDRS)]. Arquivos de neuro-psiquiatria 2005;63:119-126.

7 Chan IH, Siu AM: A study of the reliability and validity of the Chinese version of the Dementia Rating Scale. International psychogeriatrics / IPA 2005;17:69-79.

8 Chan AS, Choi MK, Salmon DP: The effects of age, education, and gender on the Mattis Dementia Rating Scale performance of elderly Chinese and American individuals. The journals of gerontology Series B, Psychological sciences and social sciences 2001;56:P356-363.

9 Chan AS, Choi A, Chiu H, Lam L: Clinical validity of the Chinese version of Mattis Dementia Rating Scale in differentiating dementia of Alzheimer's type in Hong Kong. Journal of the International Neuropsychological Society : JINS 2003;9:45-55.

10 Morris JC: The Clinical Dementia Rating (CDR): current version and scoring rules. Neurology 1993;43:2412-2414.

11 Zhang MY, Katzman R, Salmon D, Jin H, Cai GJ, Wang ZY, Qu GY, Grant I, Yu E, Levy P, et al.: The prevalence of dementia and Alzheimer's disease in Shanghai, China: impact of age, gender, and education. Annals of neurology 1990;27:428-437. 

cognitive impairment $(\mathrm{MCl})$ in medical practice: a critical review of the concept and new diagnostic procedure. Report of the $\mathrm{MCI}$ Working Group of the European Consortium on Alzheimer's Disease. Journal of neurology, neurosurgery, and psychiatry 2006;77:714-718.

13 Can H, Dogutepe E, Torun Yazihan N, Korkman H, Erdogan Bakar E: Construct Validity of Auditory Verbal Learning Test. Turk psikiyatri dergisi = Turkish journal of psychiatry 2016;27:195-203.

14 Jack CR, Jr., Albert MS, Knopman DS, McKhann GM, Sperling RA, Carrillo MC, Thies B, Phelps CH: Introduction to the recommendations from the National Institute on Aging-Alzheimer's Association workgroups on diagnostic guidelines for Alzheimer's disease. Alzheimer's \& dementia : the journal of the Alzheimer's Association 2011;7:257-262.

15 Lucas JA, Ivnik RJ, Smith GE, Bohac DL, Tangalos EG, Kokmen E, Graff-Radford NR, Petersen RC: Normative data for the Mattis Dementia Rating Scale. Journal of clinical and experimental neuropsychology 1998;20:536-547.

\section{Tables}

Table 1 Demographic information and performance of CMMSE and DRS among four groups

\begin{tabular}{|c|c|c|c|c|c|}
\hline & $N C(n=136)$ & $\mathrm{MCl}(\mathrm{n}=167)$ & $\begin{array}{l}\text { Mild AD ( } \mathrm{n}= \\
116)\end{array}$ & $\begin{array}{l}\text { Moderate AD }(n= \\
64)\end{array}$ & $\mathrm{P}$ \\
\hline Age (year) & $68.74 \pm 8.93$ & $68.81 \pm 8.30$ & $71.29 \pm 9.14$ & $70.27 \pm 9.24$ & 0.065 \\
\hline Sex (Male/Female) & $65 / 71$ & $86 / 81$ & $59 / 57$ & $34 / 30$ & 0.886 \\
\hline Education (year) & $10.73 \pm 3.89$ & $11.21 \pm 3.39$ & $10.53 \pm 4.03$ & $10.16 \pm 3.76$ & 0.214 \\
\hline CMMSE total score $(\max =30)$ & $28.20 \pm 2.10^{\star \dagger \ddagger}$ & $26.87 \pm 1.93^{\S \Phi}$ & $21.66 \pm 2.68^{\#}$ & $13.37 \pm 2.25$ & $<0.001$ \\
\hline DRS total score $(\max =144)$ & $\begin{array}{l}133.71 \pm \\
6.64^{\star \dagger \ddagger}\end{array}$ & $\begin{array}{l}128.59 \pm \\
7.03^{\S}\end{array}$ & $110.43 \pm 10.71^{\#}$ & $91.20 \pm 17.42$ & $<0.001$ \\
\hline Attention $(\max =37)$ & $36.51 \pm 0.76^{\dagger \ddagger}$ & $36.14 \pm 1.26^{\S \Phi}$ & $35.14 \pm 2.00^{\#}$ & $32.66 \pm 3.85$ & $<0.001$ \\
\hline $\begin{array}{l}\text { Initiation/Perseveration ( } \max = \\
\text { 37) }\end{array}$ & $32.50 \pm 4.12^{\dagger \ddagger}$ & $31.54 \pm 5.04^{\S \rrbracket}$ & $23.40 \pm 6.03^{\#}$ & $17.05 \pm 6.77$ & $<0.001$ \\
\hline Construction $(\max =6)$ & $5.86 \pm 0.65^{\dagger \ddagger}$ & $5.68 \pm 0.74^{\S \rrbracket}$ & $5.16 \pm 1.35^{\#}$ & $3.86 \pm 1.79$ & $<0.001$ \\
\hline Conceptualization $(\max =39)$ & $35.68 \pm 2.82^{\star \dagger \ddagger}$ & 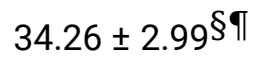 & $31.53 \pm 4.34^{\#}$ & $27.25 \pm 7.66$ & $<0.001$ \\
\hline Memory $(\max =25)$ & $23.16 \pm 2.03^{\star \dagger \ddagger}$ & 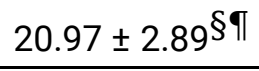 & $15.21 \pm 3.87^{\#}$ & $10.39 \pm 3.93$ & $<0.001$ \\
\hline
\end{tabular}

Notes: AD: Alzheimer's disease; MCl: mild cognitive impairment; CMMSE: mini-mental state examination-Chinese version; DRS: Mattis Dementia Rating Scale; NC: normal controls.

${ }^{*} p<0.05, \mathrm{NC}$ vs. MCl; ${ }^{\dagger} \mathrm{p}<0.05, \mathrm{NC}$ vs. Mild $A D \square^{\ddagger} p<0.05, \mathrm{NC}$ vs. Moderate $A D \square^{\S} p<0.05, \mathrm{MCl}$ vs. Mild $A D \square^{\top} p<0.05, M C l$ vs. Moderate $A D \square^{\#} p<0.05$, Mild $A D$ vs. Moderate $A D$

Table 2 ROC analyses of DRS total and subscale scores for discriminate $\mathrm{MCl}$ from $\mathrm{NC}, \mathrm{MCl}$ from mild $\mathrm{AD}$ and mild $A D$ from moderate $A D$ 


\begin{tabular}{llllllllll}
\hline & \multicolumn{3}{c}{ NC vs. MCl } & \multicolumn{3}{c}{ MCl vs. Mild AD } & \multicolumn{3}{c}{ Mild AD vs. Moderate AD } \\
\cline { 2 - 11 } & $\begin{array}{l}\text { cut- } \\
\text { off }\end{array}$ & $\begin{array}{l}\text { Sensitivity } \\
(\%)\end{array}$ & $\begin{array}{l}\text { Specificity } \\
(\%)\end{array}$ & $\begin{array}{l}\text { cut- } \\
\text { off } \\
\text { score }\end{array}$ & $\begin{array}{l}\text { Sensitivity } \\
(\%)\end{array}$ & $\begin{array}{l}\text { Specificity } \\
(\%)\end{array}$ & $\begin{array}{l}\text { cut- } \\
\text { off } \\
\text { score }\end{array}$ & $\begin{array}{l}\text { Sensitivity } \\
(\%)\end{array}$ & $\begin{array}{l}\text { Specificity } \\
(\%)\end{array}$ \\
\hline RS total score & 131 & 65.3 & 67.6 & 120 & 84.5 & 85.0 & 103 & 79.7 & 78.4 \\
ttention & 36 & 48.5 & 65.4 & 36 & 75.9 & 51.5 & 34 & 59.4 & 76.7 \\
litiation/perseveration & 30 & 38.9 & 72.1 & 29 & 87.1 & 67.7 & 16 & 50.0 & 87.9 \\
onstruction & 5 & 20.4 & 92.6 & 5 & 39.7 & 79.6 & 4 & 56.2 & 79.3 \\
onceptualization & 36 & 80.8 & 44.1 & 32 & 53.4 & 83.8 & 32 & 78.1 & 46.6 \\
lemory & 22 & 68.3 & 72.8 & 19 & 87.1 & 77.2 & 13 & 84.4 & 72.4 \\
\hline
\end{tabular}

Notes: AD: Alzheimer's disease; DRS: Mattis Dementia Rating Scale; MCl: mild cognitive impairment; NC: normal controls. 